The Be Phenomenon in Early-Type Stars, IAU Colloquium 175

ASP Conference Series, Vol. 214, 2000

M. A. Smith, H. F. Henrichs, and J. Fabregat, eds.

\title{
Origin of Circumstellar Matter in the Spectroscopic Binary CX Dra
}

\author{
Hidetoshi Iwamatsu, Ryuko Hirata, Seiji Masuda \\ Department of Astronomy, Kyoto University, Kyoto 606-8502, Japan
}

\begin{abstract}
We examine the periodic component in the polarization of CX Dra, a spectroscopic binary with an orbital period of 7 days. We find that this component originates in dense matter located in the direction preceding the secondary by about $4.5^{\circ}$ from the primary-secondary direction. This dense scattering region is attributable to the region where the $\mathrm{H} \alpha$ emission peak is formed, i.e. the gas stream or hot spot on the accretion disk of the primary. Its mass is estimated as $1.3 \cdot 10^{-11} \mathrm{M}_{\odot}$ in its well developed phase.
\end{abstract}

\section{Introduction}

The Be star CX Dra (HD174237) is a double-lined spectroscopic binary (B2.5Ve + F5III) with a period of 7 days (Horn et al. 1992). Using abundant spectroscopic material, Richards et al. (1999) have recently derived its orbital elements: $P=6.695957 \pm 0.000043$ days, $T_{\text {prim.min. }}=2442559.48 \pm 0.24 \mathrm{HJD}, e=0.052 \pm 0.006$, $q=0.23$, and $i=50^{\circ} \sim 55^{\circ}$. Koubský et al. (1998) made a radial velocity analysis of the emission peak at $\mathrm{H} \alpha$ and concluded that this emission peak orginates between the binary components in the direction preceding the secondary from the center-of-mass to the secondary direction. Huang, Hsu \& Guo (1989) observed $V$-band polarimetry of CX Dra, and found that the polarimetric variation can be divided into a periodic component with one half the orbital period and a component with relatively longer timescales. The former component can be interpreted in terms of phase-locked dense scattering matter.

We report our investigation of the circumstellar matter in CX Dra from polarimetric data. We utilized two sets of polarimetric data: V-band data obtained by Huang et al. (1989), and the weighted mean of the multi-band data obtained at the Dodaira Observatory, National Astronomical Observatory, Japan.

\section{Interpretation of the polarimetric variation}

We assume the intrinsic polarization is formed by electron scattering both in the axisymmetric disk around the primary and in local scattering material (hereafter called dense matter) fixed at some place in the binary system. The eccentricity of this system is small, and we found that its effect to the polarization in CX Dra is within the error of measurements (Brown et al. 1982). Thus, we adopt the expression given by Brown, McLean \& Emslie (1978) for $e=0$ and for a 
single scattering. We also assume that the primary is the only light source and that the circumstellar matter is symmetric with respect to the orbital plane.

Then the Stokes parameters, $q^{\prime}$ and $u^{\prime}$, with the reference vector normal to the orbital plane are described by the matrix equation:

$$
\left(\begin{array}{cc}
q^{\prime}-\tau_{0}\left(1-3 \gamma_{0}\right) \\
u^{\prime}
\end{array}\right)=\tau_{0}\left(\begin{array}{cc}
-\left(1+\cos ^{2} i\right) \gamma_{3} & \left(1+\cos ^{2} i\right) \gamma_{4} \\
-2 \cos i \cdot \gamma_{4} & -2 \cos i \cdot \gamma_{3}
\end{array}\right)\left(\begin{array}{c}
\cos 4 \pi \lambda \\
\sin 4 \pi \lambda
\end{array}\right)
$$

with

$$
\begin{aligned}
\tau_{0} & =\frac{\sigma_{0}}{2} \iiint\left\{n_{e}\right\} d R \sin \Theta d \Theta d \Phi, \\
\tau_{0} \gamma_{0} & =\frac{\sigma_{0}}{2} \iiint\left\{n_{e} \cos ^{2} \Theta\right\} d R \sin \Theta d \Theta d \Phi, \\
\tau_{0} \gamma_{3} & =\frac{\sigma_{0}}{2} \iiint\left\{n_{e} \sin ^{2} \Theta \cos 2 \Phi\right\} d R \sin \Theta d \Theta d \Phi, \\
\tau_{0} \gamma_{4} & =\frac{\sigma_{0}}{2} \iiint\left\{n_{e} \sin ^{2} \Theta \sin 2 \Phi\right\} d R \sin \Theta d \Theta d \Phi,
\end{aligned}
$$

where $i$ is the inclination angle of the orbital plane, $\lambda$ is the orbital phase, $\sigma_{0}=\frac{3 \sigma_{T}}{16 \pi}, n_{e}$ is the electron number density, and $(R, \Theta, \Phi)$ indicates the polar coordinates co-rotating with the binary. The origin of the coordinates is the center of the primary.

The transformation to the equatorial coordinates is given by

$$
\left(\begin{array}{c}
q-\bar{q} \\
u-\bar{u}
\end{array}\right)=\left(\begin{array}{rr}
\cos 2 \theta & -\sin 2 \theta \\
\sin 2 \theta & \cos 2 \theta
\end{array}\right)\left(\begin{array}{c}
q^{\prime}-\tau_{0}\left(1-3 \gamma_{0}\right) \\
u^{\prime}
\end{array}\right)
$$

where $q$ and $u$ are the observed Stokes parameters, $\bar{q}$ and $\bar{u}$ are those originating in the axisymmetric disk. The quantity $\theta$ is the angle between the direction to the north pole and the vector normal to the orbital plane projected on the sky. The quantities $\bar{q}$ and $\bar{u}$ can be estimated from the mean values at each epoch.

\section{Position of the dense scattering matter}

The line fitted through the data passes through the zero point in the $(q, u)$ plane at a polarization angle $80^{\circ}$. Thus, the direction of the axisymmetric disk is $10^{\circ}$
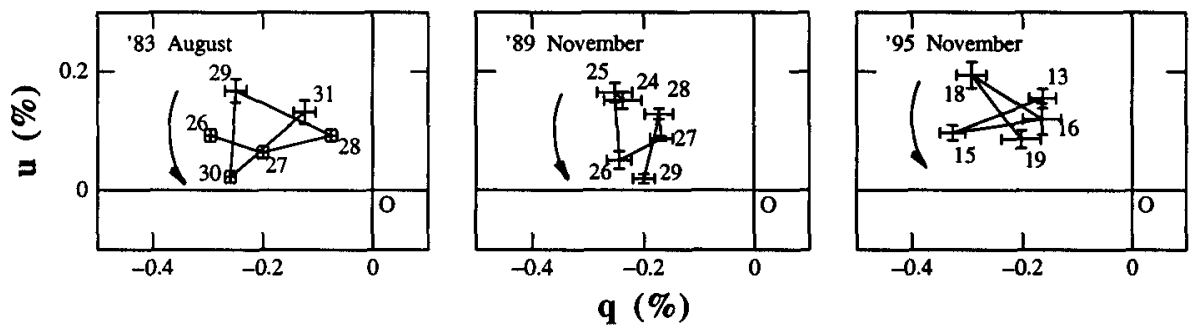

Figure 1. The variation on $(q, u)$ plane in three epochs. All indicate that the observing point rotates counter-clockwise. 
westwards, measured from the equatorial north. The interstellar polarization towards CX Dra is either negligibly small or its direction is parallel or antiparallel to the intrinsic polarization. The polarization of nearby stars is very small. We therefore conclude that the interstellar polarization can be neglected towards CX Dra.

Figure 1 illustrates the observed variation in the $q-u$ plane at three epochs. One can see that the binary rotates counter-clockwise. We found that the variation of the periodic component is larger when the background (averaged) polarization is larger. This indicates that the dense matter also develops when the disk develops. From three observing epochs in 1983, we also confirmed that the averaged polarization became larger when the $\mathrm{H} \alpha$ emission intensity was larger.

Adopting $i=53.5^{\circ}$ (Richards et al. 1999), and inserting the quantities $q-\bar{q}$, and $u-\bar{u}$ into equation(3), we obtained the following best-fitted parameters: $\tau_{0} \gamma_{3}=0.040 \pm 0.002$ and $\tau_{0} \gamma_{4}=0.006 \pm 0.002$ (Figure 2a).

Assuming that the dense matter is located at $\left(R_{0}, 0, \Phi_{0}\right)$ with a constant electron density, $\tau_{0} \gamma_{3}$ and $\tau_{0} \gamma_{4}$ can be approximated as

$$
\tau_{0} \gamma_{3} \simeq \frac{\sigma_{0}}{2 R_{0}^{2}} n_{e} V \cos 2 \Phi_{0}, \quad \tau_{0} \gamma_{4} \simeq \frac{\sigma_{0}}{2 R_{0}^{2}} n_{e} V \sin 2 \Phi_{0},
$$

where $R_{0}$ is the distance between the primary and the dense matter, and $V$ is its volume. Then we have

$$
\Phi_{0}=\frac{1}{2} \arctan \frac{\tau_{0} \gamma_{4}}{\tau_{0} \gamma_{3}}=4.5 \pm 1.5^{\circ} .
$$

Thus, the dense matter is located towards the direction preceding the secondary by about $4.5^{\circ}$ from the primary-secondary direction. Figure $2 \mathrm{~b}$ shows the
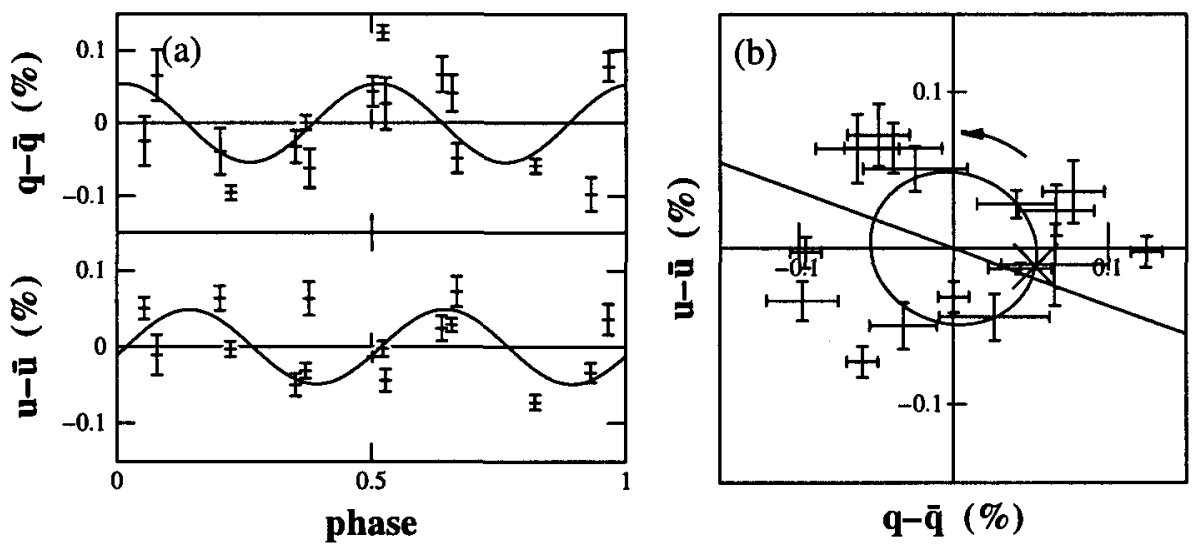

Figure 2. (a): A sinusoidal fit for $q-\bar{q}$ and $u-\bar{u}$. (b): Observed points rotate counter-clockwise. The point "X" corresponds to phase zero. The polarization from the axisymmetric disk lies on the straight line indicated. 
variation of the periodic component in the $(q, u)$ plane, with the fitted ellipse. It is likely that the dense region determined polarimetrically is identical to the region where the $\mathrm{H} \alpha$ emission peak is formed (Koubský et al. 1998, Richards et al. 1999). We assume $R_{0}=13 \mathrm{R}_{\odot}$ from the $\mathrm{H} \alpha$ analysis. Then we have

$$
n_{e} V=\frac{2 R_{0}^{2}}{\sigma_{0}} \sqrt{\left(\tau_{0} \gamma_{3}\right)^{2}+\left(\tau_{0} \gamma_{4}\right)^{2}}=(1.52 \pm 0.09) \cdot 10^{46}
$$

Its mass is estimated as $(1.3 \pm 0.1) \cdot 10^{-11} \mathrm{M}_{\odot}$ under the assumption of fully ionized hydrogen. The amplitude of the periodic component at epochs with small averaged polarization is estimated to be about one half compared to that at epochs with large averaged polarization, indicating that its mass is also one half the quoted value.

If the dense matter is taken as a uniform sphere, we can calculate the radius $R$ and optical depth $\tau_{\mathrm{e}}$ for electron scattering by assuming the electron number density: $R=4.8 \mathrm{R}_{\odot}$ and $\tau_{\mathrm{e}}=0.0022$ for $n_{\mathrm{e}}=10^{11}\left(\mathrm{~cm}^{-3}\right), R=2.2 \mathrm{R}_{\odot}$ and $\tau_{\mathrm{e}}=0.10$ for $n_{\mathrm{e}}=10^{12}$, and $R=1.0 \mathrm{R}_{\odot}$ and $\tau_{e}=0.47$ for $n_{\mathrm{e}}=10^{13}$.

\section{Conclusion}

1. The CX Dra binary system rotates counter-clockwise on the projected sky, and the direction of the orbital plane is $10^{\circ}$ westwards, measured from the equatorial north.

2. An accumulation of dense matter is located in the direction preceding the secondary by about $4.5^{\circ}$ from the primary-secondary direction. Thus, we see the gas stream from the secondary or the hot spot or its vicinity as the dense matter. This is consistent with the result of $\mathrm{H} \alpha$ analysis (Šimon 1996, Koubský 1998, Richards et al. 1999). The total mass of the dense matter is about $10^{-11} \mathrm{M}_{\odot}$ in its well developed epoch.

3. The periodic component in polarization degree increases as the background (averaged) polarization degree increases. Thus, the mass of the dense matter increases as the disk develops. The polarization degree seems to increase with the increase of the $\mathrm{H} \alpha$ emission intensity.

\section{References}

Brown, J.C., McLean, I.S., Emslie, A.G. 1978, A\&A 68, 415

Brown, J.C., Aspin, C., Simmons, J.F.L., McLean, I.S. 1982, MNRAS 198, 787

Huang, L., Hsu, J.C., Guo, Z.H. 1989, A\&AS 78, 431

Horn, J., Hubert, A. M., Hubert, H., Koubský, P., Bailloux, N. 1992, A\&A 259, L5

Koubský, P., et al. 1998, Hvar Obs. Bull. 22, 1, 17

Richards, M.T., Koubský, P., Šimon, V., Peters, G. J., Hirata, R., Škoda, P. \& Masuda, S. 1999, ApJ, in press

Šimon, V. 1996, A\&A 308, 799 